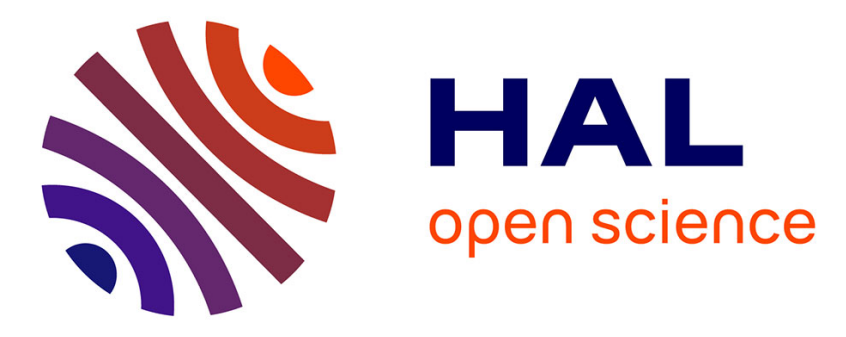

\title{
Multi-parasite host susceptibility and multi-host parasite infectivity: A new approach of the Biomphalaria glabrata/Schistosoma mansoni compatibility polymorphism.
}

\author{
A. Theron, A. Rognon, Benjamin Gourbal, G. Mitta
}

\section{To cite this version:}

A. Theron, A. Rognon, Benjamin Gourbal, G. Mitta. Multi-parasite host susceptibility and multi-host parasite infectivity: A new approach of the Biomphalaria glabrata/Schistosoma mansoni compatibility polymorphism.. Infection, Genetics and Evolution, 2014, 26, pp.80-88. 10.1016/j.meegid.2014.04.025 . halsde-00998747

\section{HAL Id: halsde-00998747 https://hal.science/halsde-00998747}

Submitted on 2 Jun 2014

HAL is a multi-disciplinary open access archive for the deposit and dissemination of scientific research documents, whether they are published or not. The documents may come from teaching and research institutions in France or abroad, or from public or private research centers.
L'archive ouverte pluridisciplinaire HAL, est destinée au dépôt et à la diffusion de documents scientifiques de niveau recherche, publiés ou non, émanant des établissements d'enseignement et de recherche français ou étrangers, des laboratoires publics ou privés. 
Multi-parasite host susceptibility and multi-host parasite infectivity: a new approach of the Biomphalaria glabrata / Schistosoma mansoni compatibility polymorphism

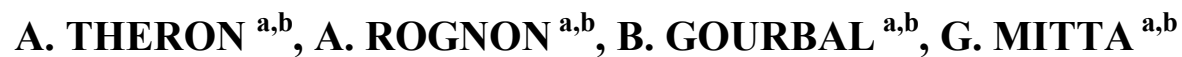

${ }^{\text {a }}$ CNRS, UMR 5244, Ecologie et Evolution des Interactions (2EI), Université de Perpignan, France.

b Université de Perpignan Via Domitia. 52, Ave Paul Alduy. 66860 Perpignan Cedex, France.

Corresponding author. Tel.: + 33468662183 ; fax: + 33468502281

E-mail address: theron@univ-perp.fr (A. Theron)

\begin{abstract}
In this study, we analyze the degree of susceptibility/un-susceptibility of five strains of Biomphalaria glabrata from different geographical origins successively challenged with a panel of 4 Schistosoma mansoni strains. A total of 20 homopatric and heteropatric hostparasite combinations were tested with exposure doses of 1, 10, 20, 30 and 50 miracidia per individual host. By doing this, we characterized each B. glabrata strain by its "multi-parasite susceptibility phenotype" that reflects better the efficiency of their defense mechanism against not only one, but a diversity of schistosome stocks. In the same time, all the S. mansoni strains used were characterized, by their "multi-host infectivity phenotype" that reflects the level of infectivity they display when confronted to diverse snail populations. Based on these results it is possible to select different homogenous stocks of snails with different spectrum of susceptibility/un-susceptibility for several parasite strains. This will be a useful tool for future functional studies conducted to understand the genetics and molecular basis of the compatibility polymorphism in this host/parasite model.
\end{abstract}

Keywords:

Schistosoma mansoni

Biomphalaria glabrata

Compatibility polymorphism

Matching phenotypes 


\section{Introduction}

Schistosomiasis remains an important health problem that affects over 200 million people worldwide (WHO, 2010). Schistosoma mansoni causes intestinal schistosomiasis and as all trematode species, needs mollusks as first intermediate hosts to accomplish part of its life cycle before infecting humans. For this species, only fresh water snails of the genus Biomphalaria are potentially susceptible to an infection by the larval stages of the parasite. However, within a same snail species, for example B. glabrata, the main intermediate host of S. mansoni in South America, compatibility varies between populations, strains or individuals (Basch, 1976; Theron et al, 1997). Snail/schistosome compatibility reflects both snail susceptibility and schistosome infectivity and the observed polymorphism of compatibility results from the complex phenotype-by-phenotype interactions occurring between each individual host and each individual parasite (Basch, 1976; Theron and Coustau, 2005; Theron et al., 2008). The immunologic mechanisms underlying compatibility have remained largely unknown (see Bayne, 2009; Yoshino and Coustau, 2011). However recent studies have succeed in identifying host immune recognition receptors and parasite antigens displaying a high level of variability and which were described to be in interaction (Roger et al., 2008; Moné et al., 2008, 2010). It was hypothesised that these molecules could be involved in the expression of the B. glabrata / S. mansoni compatibility polymorphism (Mitta et al., 2012; Hanington et al., 2012; Roger et al., 2008a,b,c; Moné et al., 2010; 2011).

Different B. glabrata laboratory strains (or isolates) show various degrees of susceptibility to S. mansoni infection (Basch, 1976; Theron et al., 1997) and different strains of S. mansoni display different levels of infectivity towards a particular strain of snail host. Compatibility is strain specific (Theron and Coustau, 2005; Webster and Davies, 2001), one snail strain that is highly susceptible to one particular strain of schistosome could be partially or totally unsusceptible to another schistosome strain (Richards, 1984; Lie et al., 1979). On the other hand, one parasite strain that is highly infective to a snail host strain could be less or un-infective to another one. In spite of this overview about variation of compatibility, it appears that very few studies have investigated the multi-parasite strain susceptibility of $B$. glabrata stocks and conversely the multi-host strain infectivity of $S$. mansoni stocks.

The aim of the present study was to characterize and compare the degree of susceptibility/un-susceptibility of different strains of B. glabrata when challenged with a panel of $S$. mansoni strains. On the other hand, the infectious capacity of the different strains of $S$. mansoni used was also evaluated when confronted to a panel of B. glabrata stocks. By 
this way we will be able to discriminate particular strains of snails that are more or less efficient in their defense mechanisms against not only a single strain of parasite but against a diversity of schistosome strains and then, to go beyond the classic approach that traditionally characterize a strain either resistant or susceptible. This approach, concerning both the hosts and the parasite may lead to the selection of snail phenotypes representative of different spectrum of susceptibility/un-susceptibility and schistosome strains representative of different capacities of infectivity. Such discreet phenotypes of multi-parasite susceptibility provide an invaluable resource for future comparative genetics and functional studies to better understand the genetics and molecular basis of the compatibility polymorphism in this host/parasite model.

\section{Materials and methods}

\subsection{Parasites and snails}

Four strains of $S$. mansoni (Sm) from different geographical origins were used in this study. Each was maintained in the laboratory since more than 10-20 years on their sympatric strains of B. glabrata (Bg) as intermediate host and in mice (SWISS OF1) as definitive host. These four homopatric host/parasite combinations are termed as follow: BgVEN / SmVEN (origin Venezuela, Guaraca); BgGUA / SmGUA (origin Guadeloupe, Dans Fond); BgBRE / SmBRE (origin Brazil, Recife) and BgBAR / SmLE (origin Brazil, Belo Horizonte). One supplementary B. glabrata strain for which we do not dispose of its corresponding parasite strain was also used: the BgBS-90 strain (origin Brazil, Salvador).

\subsection{Compatibility trials}

As snail infection rates vary with the parasite dose (Theron et al., 1997; 2008), compatibility tests were conducted by dose-response curves obtained by challenging individual snails (35-40 snails of 6-8 $\mathrm{mm}$ in diameter per treatments) with doses of 1, 10, 20, 30 and 50 freshly hatched miracidia (Mi) in $5 \mathrm{ml}$ of water at $24-25^{\circ} \mathrm{C}$ for $8 \mathrm{~h}$. Following exposure to miracidia, snails were replaced in their original containers until their infection status was assessed. For all experiments, infection of snails was evaluated by the presence and count of mother sporocysts at 15 days post-exposure as previously described (Theron and Gerard, 1994; Allienne et al., 2011). Briefly summarized, snails were relaxed in pond water 
containing excess of crystaline menthol for $12 \mathrm{hr}$. The snail body was removed and fixed in modified Raillet-Henry solution. Using this technique, mother sporocysts were readily observable as translucent white bodies within an opaque brown tissue background. The number of developped mother sporocysts (containing young daughter sporocysts) present within each snail was determined following exhaustive dissection of the snail including deeper tissues. A previous histological study on BgGUA snails exposed to SmGUA strain and fixed $48 \mathrm{hr}$ later, showed that among the miracidia that have penetrated, 22\% appeared well developped without host reaction, $40 \%$ were encapsulated by the host hemocytes and $38 \%$ degenerated without host reaction (Theron et al., 1997). One hour after exposure, $26 \%$ of the miracidia had not penetrated the snail. The level of compatibility was quantified by the proportion of infected snails and the intensity of infection by the number of developped mother sporocysts (MSp) per snails. All the host-parasite combinations identified as compatible by the presence of developing mother sporocysts at 15 days post-infection, were able to produce cercariae. This has been verified during the laboratory cycling of the various strains of $S$. mansoni on the different strains of snails and additionaly, during the experimental selection of compatible phenotypes of snail hosts currently in progress.

For each of the 5 snail strains, compatibility was tested separately with their homopatric schistosome strain and the 3 other heteropatric parasite strains respectively (for the BS-90 snail strain, the 4 parasite strains used were heteropatric). In total, the following 20 host-parasite combinations were tested with exposure doses of 1, 10, 20, 30 and 50 miracidia per host : (BgBAR / SmLE, BgBAR / SmVEN, BgBAR / SmBRE, BgBAR / SmGUA); (BgVEN / SmVEN, BgVEN / SmLE, BgVEN / SmBRE, BgVEN / SmGUA); (BgBRE / SmBRE, BgBRE / SmLE, BgBRE / SmVEN, BgBRE / SmGUA); (BgGUA / SmGUA, BgGUA / SmLE, BgGUA / SmVEN, BgGUA / SmBRE); (BgBS-90 / SmGUA, BgBS-90 / SmLE, BgBS-90 / SmVEN, BgBS-90 / SmBRE).

\section{Results}

\subsection{Variations of susceptibility between B. glabrata strains (Fig. 1)}

For all the host/parasite combinations tested, the dose-response curves generated showed the same pattern: a rapid increase in infection rates between the doses 1 and 10-20 miracidia per snail then a plateau that leveled at different rates (Fig. 1). 
Each snail strain exhibits a particular scope of susceptibility when confronted to the 4 strains of schistosomes (Fig. 1). The BgBRE strain was 100\% susceptible with 3 (SmLE, SmVEN and SmBRE) of the 4 schistosome strains tested but very poorly susceptible to the SmGUA (around 5\%). At the opposite, BgBAR strain, 100\% susceptible with its sympatric schistosome strain (SmLE), was only 50\% susceptible with SmVEN, 10\% with SmBRE and totally unsusceptible to SmGUA. BgVEN and BgGUA strains showed a similar pattern of susceptibility (100\% with SmLE and SmVEN, 80\% with SmBRE and 40\% with SmGUA). The BgBS-90 stock was only partially susceptible to the SmLE strain of S. mansoni (53\% of infection with $20 \mathrm{Mi}$ ) and totally unsusceptible to the 3 other strains of $S$. mansoni.

Excepted for the homopatric couple BgGUA/SmGUA that showed the lowest compatibility level compared to the corresponding heteropatric combinations, the 3 other homopatric couples (BgBRE/SmBRE, BgVEN/SmVEN and BgBAR/SmLE) were equally or more compatible than their corresponding heteropatric couples.

\subsection{Variations of infectivity between S. mansoni strains (Fig. 2)}

By the side of the parasite, clearly the SmLE strain appeared as having the highest capacity of infection whatever the snail strain used. Infection rates of $100 \%$ were obtained with the dose of 10-20 Mi per snail with 4 snail strains BgBRE, BgGUA, BgVEN and BgBar. SmLE was the only schistosome strain tested capable of infecting the BgBS-90 strain (53\% of infection with 20Mi). At the opposite, the SmGUA strain appeared as the less infective even towards its sympatric host snails (40-50\% of BgGUA infected at the plateau), equivalent infection rates were obtained only with the BgBAR stock. SmGUA was very few infective for BgBRE (around 5\%) and un-infective towards the BgBAR and BgBS90 strains. SmVEN and SmBRE strains showed high level of infectivity with the BgBRE (100\%), BgGUA (80\%) and BgVEN (80\%) snail stocks, low with the BgBAR strain (15\%) and un-infective towards the BgBS-90 strain.

\subsection{Variations of parasite intensities (Fig. 3, A, B)}

Variations of parasite intensities (number of developed MSp per snails), are presented in figure 3 only for the BgBRE host strain challenged by the 4 parasite strains and for the SmBRE parasite strain infecting the 5 host strains at the different doses of miracidia. These two examples were representative of the other combinations. For compatible combinations, 
the number of MSp increases with the miracidial doses at exposure. For the homopatric couple SmBRE/BgBRE (Fig. 3 A) the mean number of MSp /snail for $10 \mathrm{Mi}$ was of $3.58 \pm$ 0.19 and raised to $16.18 \pm 0.86 \mathrm{MSp}$ for the dose of $50 \mathrm{Mi} /$ snail. For the less compatible combination, SmBRE/BgBAR (Fig. 3 B) the number of MSp remained stable around 2.5 whatever the miracidial dose was.

For the same parasite strain and at equivalent dose of Mi, variations exist as a function of the host strain challenged and its level of susceptibility. Highest was the compatibility level, highest was the mean parasite intensity. As an example (Fig. 3 B), the relationship between mean parasite intensities and compatibility level for the SmBRE strain at the dose of $50 \mathrm{Mi} /$ snail infecting the 4 host strains was as follow: $2.5 \mathrm{MSp}$ and $16 \%$ of infection for SmBRE/BgBAR, $8.0 \mathrm{MSp}$ and $75 \%$ for SmBRE/BgVEN, $10.0 \mathrm{MSp}$ and $90 \%$ for SmBRE/BgGUA, 16.0 MSp and 100\% for SmBRE/BgBRE.

We note that only a low proportion of miracidia presented to the snail host succeed in developing to mother sporocysts and this proportion varies between parasite strains, host strains and miracidial doses at exposure (Fig. 4). Including data from all the host strains and all the miracidial doses, SmLE parasite strain showed the highest Mi/MSp transformation rate with an average of $35 \%$, SmGUA the lower (20\%). Including data from all the parasite strains and the miracidial doses, it is among the snails of the BgBRE strain that we observed the highest rate of developing miracidia (35\%) and among the snails of the BgBAR strain the lowest (16\%).

\section{Discussion}

Investigations on snail/schistosome compatibility between Biomphalaria species and S. mansoni started several decades ago with the pioneer works of Files and Cram (1949), Abdel-Malek (1950), then Richards (1970, 1973), and Basch (1976). Later, numerous studies confirmed strong variations in host susceptibility or parasite infectivity, depending of the geographic origin of the host and parasite strains used (Lie et al., 1979; Richard and Shade, 1987; Morand et al., 1996; Webster et al., 2001). Our study addressed another component of this snail/schistosome compatibility polymorphism to the extent that the susceptibility / unsusceptibility level of different strains of B. glabrata was determined when confronted to the same panel of different S. mansoni strains. By doing this, we were able to characterize each B. glabrata strain by what we defined as its "multi-parasite susceptibility phenotype" and we may better compare the efficiency of their defense mechanism against not only one, 
but a diversity of schistosome stocks. In the same time, we characterized each of the $S$. mansoni strain used by their "multi-host infectivity phenotype" that reflects the level of infectivity they display when confronted to a diversity of snail stocks. Clearly the results obtained, showed marked differences between both host and parasite strains in their capacity to resist or infect the diversity of parasites or hosts proposed, respectively. This offers the possibility to select experimentally new homogeneous susceptible/unsuceptible phenotypes of snails for further studies.

\subsection{A matching phenotype-phenotype interaction}

As previously demonstrated for one of the host/parasite combination tested in this study (i.e. SmGUA/BgGUA, see Theron et al., 2008), we note that all the dose-response curves obtained for each of the 20 host/parasite couples analyzed showed the same shape. They are characterized by a rapid increase of the infection rates between 1 and 10-20 miracidia per snail, then an asymptote that leveled off at different values as a function of the strain used. For combinations with compatibility level lower than $100 \%$, the parasite intensities increased with increasing miracidial doses but not the proportion of infected hosts (at 20,30 and $50 \mathrm{miracidia} / \mathrm{snail}$ ). This may indicate that there is no threshold dose for which the parasite would be able to overwhelm the host defenses. Similar pattern of dose-response curves with the plateau that leveled below $100 \%$ were obtained with higher miracidial doses (80 and $100 \mathrm{Mi} /$ snail, data not shown). Increasing the miracidial dose simply involves sampling a larger fraction of the phenotypic diversity in the parasite strain. In other words, a dose of 20 miracidia per snail contains, for all the $S$. mansoni strains, most of the possible phenotypes that potentially match with the host phenotype challenged. These results agree with the hypothesis that the success or failure of a challenge by one parasite mainly depends on the matched/mismatched status of the host and parasite phenotypes (Bash, 1975; Theron and Coustau, 2005; Theron et al., 2008; Mitta et al., 2012). Taking into account the little water volume used $(5 \mathrm{ml})$ for the exposition of individual snails to the miracidia, we assume that all miracidia had contact with the host. Despite this high host/parasite contact, only a small proportion of miracidia (between 20 and 40\%) is not recognized by the innate defense system of the host and develops into Msp, the other larvae are encapsulated or degenerated (Theron et al., 1997). This means that within the same snail host, a specific defense response occurs against some incompatible entered miracidia but without effect on neighboring compatible larvae. The reason of the observed different fates of the nondeveloping MSp 
(encapsulated vs degenerated) has until now received little attention. The absence of hemocytic reaction around the degenerated MSp strongly suggests unidentified humoral factors responsible for the nondevelopment of these larvae (Sullivan and Richards, 1981; Vasquez and Sullivan, 2001). Such humoral reaction against MSp was always observed in case of immune priming of B. glabrata (Sire el al., 1998; Portela et al., 2013). Indeed, MSp of the challenge infection of B. glabrata degenerate progressively within the host tissues without cellular encapsulation. Then, in case of "simultaneous" pluri-miracidial exposure (in our case 8 hours of exposition), not all miracidia penetrate the snail in the same time and we could hypothesize that incompatible miracidia penetrating first and recognized as non-self should be rapidly encapsulated while those penetrating later after this primary hemocyte-mediated defense should be stopped in their development by the presence of toxic plasma molecules and died undergoing gradual degeneration. Physiological unsuitability of the intermediate host was also proposed as a possible factor for the nondevelopment of some MSp (Vasquez and Sullivan, 2001). As emphasized by Basch (1976), compatibility is tested independently for each entering miracidium and each exposed snail. The phenotype (susceptible vs unsusceptible) of a host is expressed as a function of the parasite genotype it harbors and reciprocally, the phenotype (infective vs uninfective) of a parasite is expressed as a function of the genotype of the particular host that it enters.

\subsection{Variations in host susceptibility and parasite infectivity}

According to the pattern of the dose-response curves, the value of the infection rates at the plateau for $20 \mathrm{Mi} /$ snail was retained to define and compare the level of susceptibility/unsusceptibility between the snail/schistosome combinations studied and a radar graph presentation will facilitate such comparisons (Fig. 5 and 6). By the side of the host strains (Fig. 5), a gradient of increasing multi-parasite susceptibility could be established. Snails of the BS90 strain appeared as those having the best efficient internal defense system against the four strains of $S$. mansoni tested. However we note that within this highly un-susceptible strain, that was historically considered as "totally resistant" to both new and old world $S$. mansoni strains (Ittiprasert and Knight, 2012) half of the B. glabrata snails was unable to eliminate $S$. mansoni of the SmLE strain. The BgBAR strain, unsusceptible to SmGUA is poorly susceptible to SmBRE and SmVEN, but totally susceptible to SmLE. The BgBRE strain is $100 \%$ susceptible to three parasite strains but highly unsusceptible to SmGUA. BgGUA and BgVEN strains show similar pattern and appear as the most susceptible to all 
parasite strains. None of the snail strains used was totally (100\%) susceptible to the four $S$. mansoni strains respectively (Fig. 5).

Marked variations are also observed as related to the infectivity spectrum displayed by the parasite strains, ranging from the SmGUA poorly infective or un-infective towards the 5 host strains, to SmLE that is able to infect $100 \%$ of the four host strains and half of the BgBS90 snails (Fig. 6). Except for the BgGUA/SmGUA combination (37\% of infection), all the other homopatric host/parasite couples showed $100 \%$ of compatibility. Among the homopatric combinations it is interesting to note that for the snail stock BgBAR that yields the highest multi-parasite un-susceptibility level, its corresponding parasite strain (SmLE) displays on the contrary the highest multi-hosts infectivity level. Such negative correlation between host susceptibility and parasite infectivity was however not so apparent for the other snail/schistosome homopatric couples.

We must emphazized that all the S.mansoni and B. glabrata strains used in this study were maintained in the laboratory during several years and their infectivity and susceptibility phenotypes are not representative of the corresponding natural populations (Theron et al., 2008; Bech et al., 2010). However data collected during many years of routine maintenance of $S$. mansoni strains in our laboratory do not show significant change in their compatibility phenotypes after their establishment (personal observation). More precisely, previous molecular analyses using 15 microsatellite markers carried out on the SmGUA/BgGUA combination, have revealed that a loss of genetic diversity occured gradually from the field $S$. mansoni isolate to the last laboratory generation analysed after three years of cycling (Bech et al., 2010). What was interesting is the fact that, while the compatibility level decreases sharply between the field isolate and the first laboratory generation, later the degree of compatibility does not evolve and remains at the same level during all the successive laboratory generations despite repeated bottlenecks at each maintenance cycle. Then, a substantial level of compatibility polymorphism can be maintained independently of the decrease of neutral genetic diversity (Bech et al., 2010). Such phenomenon could be linked to recently described mechanisms able to generate high diversification levels in polymorphic antigens of S. mansoni (Mitta et al., 2012) through somatic recombination events and regulatory mechanisms occuring during their expression (see below) .

\subsection{Possible underlying molecular mechanisms}


The co-evolution dynamics between hosts and parasites involves huge reciprocal selective pressures on both protagonists. This co-evolutionary dynamics leads to an arms race expected to shape molecular determinants playing a key role at the core of the interaction. Relatively few reports have evaluated the impact of these reciprocal antagonistic pressures on the molecules involved in host defense mechanisms and parasite infectivity. Recent molecular approaches of the mechanisms underlying this compatibility polymorphism have identified a group of polymorphic antigens (the SmPoMucs ) expressed by the miracidia of S. mansoni that interact with polymorphic and/or diversified immune recognition molecules (the FREPs) of B. glabrata (Roger et al., 2008a, b, c; Moné et al., 2011; Hanington et al., 2012; Mitta et al., 2012). Co-evolutionary dynamics could also play on effector pathways as we demonstrated a reciprocal adaptation between host reactive oxygen species (ROS) and parasite ROS scavenger traits (Moné et al., 2011). Indeed, comparison between two homopatric couples of $B$. glabrata/S. mansoni with different compatibility level showed that higher ROS-production capacity by the host was associated with a higher ROS-scavenging ability by the parasite (highly compatible couple) and in contrast, lower ROS production by the host with lower ROS-scavenging by the parasite (less compatible combination).

Then compatibility polymorphism in B. glabrata/S. mansoni interaction could be the result of multigenic and "multi-mechanism" processes in which the relative weight of recognition mechanisms of the host and antigenic diversity of the parasite could be in balance with the effector ability (ROS production) of the host and anti-effector mechanism (ROS scavengers) of the parasite. This hypothesis could be tested in the different strains used in the present study by analysing the polymorphism occurring on FREPS and SmPoMuc to evaluate the investment of each strain in these recognition/antigenic variation processes. We could also envisage more global transcriptomic or proteomic approaches to take into account the mechanism variability we can have during co-evolutionary processes between these different populations. This would be facilitated by the use of discreet host phenotypes selected for their spectrum of susceptibility/un-susceptibility towards several strains of S. mansoni.

\subsection{Selection of multi-parasite (un)-susceptible host phenotypes}

A snail strain is not homogenous but composed of multiple host phenotypes in respect of their susceptibility/un-susceptibility towards the diversity of parasite phenotypes, then, the distribution of these phenotype frequencies within a host strain may be modified by selection. Most of the studies on variation of snail susceptibility were interested in experimental 
selection of host stocks yielding either $0 \%$ or approaching $100 \%$ infection frequencies when exposed to only one particular strain of schistosome (Mascara et al., 1999; Richards et al., 1992; Webster and Woolhouse, 1998). As we know that a host strain un-susceptible to one parasite strain could be totally susceptible to another one, such an approach gives limited information about the real efficiency level of its defense system against the diversity of parasite phenotypes. Based on the results we obtained by exposing different snail strains to different strains of schistosomes, the new idea would be to select different stocks of snails showing different spectrum of susceptibility/un-susceptibility vis-à-vis of several parasite strains. The figure 7 shows how selection patterns could be operated on the different strains of B. glabrata used in order to produce homogenous snail stocks of different multi-strain (un)susceptibility types. Within the BgBS-90 strain, by successive elimination of the snails susceptible to SmLE, we can select a snail stock with a phenotype (A) characterized by its high defense efficiency since capable to eliminate four different strains of schistosome. Eliminating snails susceptible to SmBRE and SmVEN within the BgBAR strain, we can select another host phenotype (B), un-susceptible to three parasite strains (SmVEN, SmGUA and SmBRE). Within both, BgGUA and BgVEN strains, elimination of snails susceptible to SmGUA and SmBRE allows creating two snail stocks of phenotype (C) both un-susceptible to two parasite strains. In the same way using the BgBRE strain and by successive elimination of the few snails susceptible to SmGUA, we can select another phenotype (D) un-susceptible to only one strain of schistosome. Finally with snails of the BgGUA and BgVEN, but in this case by increasing the frequence of snails susceptible to both SmGUA and SmVEN, we will select a phenotype (E) with the less efficient defense system since totally susceptible to the four different strains of schistosomes.

\section{Conclusion}

Our study through reciprocal cross-infections between five strains of B. glabrata and four strains of $S$. mansoni (i.e. 20 different homopatric and heteropatric host-parasite combinations) confirms the high degree of compatibility polymorphism that occurs in this host/parasite system. Differences in dose-response curves that characterize each snail stock (i.e. increase rate of infection at low miracidial doses then level of the asymptote) could result from the variations of the number and frequence of compatible combinations within each strain of B. glabrata when confronted to the different strains of schistosomes. 
By using the same panel of parasite strains against each snail stock, our results demonstrate that some $B$. glabrata strain have defense mechanisms more efficient than other strains through probably a better capacity to recognize a larger diversity of parasite phenotypes present within each schistosome strains and perhaps also a higher effector ability in ROS production. Thus depending on the genotypic diversity in the host and parasite isolates, the snail-schistosome compatibility would be determined by a combination of changes and a balance in recognition and effector mechanisms that determine the success or failure of the infection.

The compatibility polymorphism exists not only at this inter-strain level but also between individual hosts of a same strain. A snail population is not homogenous with regard to the susceptibility to schistosome miracidia. Within a strain, each individual host possesses its specific capacity of defense against all (unsusceptibility) or solely a part (susceptibility) of the phenotypic diversity of the miracidia from a parasite strain. The same diversity exists also for the parasite strains with some of then being more infective toward the panel of B. glabrata stock than other used in this study. Each parasite strain discriminates between the different individual host phenotypes of a snail strain. This variation of susceptibility between and within snail strains gives the opportunity, through experimental directional selection, to develop new and more homogenous snail stocks differing by their multi-parasite (un-) susceptibility phenotype (i.e., the number of schistosome strains with which there are not partially, but totally (un-)compatible). This will be a useful tool for future comparative functional studies conducted on different effectors mechanisms or recognition processes involving other immune receptors or antigens to better understand the genetics and molecular basis of the compatibility polymorphism in this host/parasite model.

To conclude and as previously mentioned, we must keep in mind that levels of compatibility characterizing laboratory strains are not representative of their corresponding field populations (Theron et al., 2008; Bech et al., 2010). Then we should proceed with caution to the possibility of extrapolating results of such studies in order to discuss epidemiological implications as for example, geographical compatibility variations and colonization risk of new areas or disease spreading that might occur under effects of climate and global changes (Morley, 2011). However, such laboratory studies on a diversity of parasite and host strains, provide detailed informations on molecular determinants playing a key role in the host-parasite compatibility interaction. This may contribute to the search for molecular markers which might inform on the potential efficiency of the defense system of 
snails from a wild population and on the infectivity capacity of a schistosome population towards snail hosts .

\section{Acknowledgements}

The authors are indebted to E. S. Loker (Center for Evolutionary and Theoretical Immunology, University of New Mexico, Albuquerque, USA) for providing the BS-90 stock of snails, to Diana Ballen (Instituto Venezolano de Investigaciones Científicas (IVIC), Caracas, Venezuela) for the snails and schistosomes from Venezuela and G. Oliviera (Instituto Nacional de Ciência e Tecnologia em Doenças Tropicais, Fundacao Oswaldo Cruz, Brazil) for the BgBAR and SmLE strains. This work was funded by the ANR (grant \# 25402 BiomGenIm; ANR-07-BLAN-0214-03), the CNRS, and the UPVD. The funders had no role in the study design, data collection, data analysis, decision to publish, or preparation of the manuscript. 


\section{References}

Abdel-Malek, E.T., 1950. Susceptibility of the snail Biomphalaria boissyi to infection with certain strains of Schistosoma mansoni. Am. J. Trop. Med. Hyg. 30, 887-894.

Allienne, J.F., Theron, A., Gourbal, B., 2011. Recovery of primary sporocysts in vivo in the Schistosoma mansoni / Biomphalaria glabrata model using a simple fixation method suitable for extraction of genomic DNA and RNA. Exp. Parasitol. 129, 11-16.

Basch, P.F., 1975. An interpretation of snail-trematode infection rates: specificity based on concordance of compatible phenotypes. Int. J. Parasitol. 5, 449-452.

Basch, P.F., 1976. Intermediate host specificity in Schistosoma mansoni. Exp. Parasitol. 39, 150-169.

Bayne, C.J., 2009. Successful parasitism of of vector snail Biomphalaria glabrata by the human blood fluke (trematode) Schistosoma mansoni: A 2009 assessment. Mol. and Bio. Parasitol. 168, 8-18.

Bech, N., Beltran, S., Portela, J., Rognon, A., Allienne, J-F., Boissier, J., Theron, A., 2010. Follow-up of the genetic diversity and snail infectivity of a Schistosoma mansoni strain from field to laboratory. Inf. Genet. Evol. 10, 1039-1045.

Files, V.S., Cram, E.B., 1949. A study on the comparative susceptibility of snails vectors to strains of Schistosoma mansoni. J. Parasitol. 35, 555-560.

Hanington, P.C., Forys, M.A., Loker, E.S., 2012. A somatically diversified defense factor, FREP3, is a determinant of snail resistance to schistosome infection. PLoS Negl. Trop. Dis. 6:e1591.

Ittiprasert, W., Knight, M., 2012. Reversing the resistance phenotype of the Biomphalaria glabrata snail host Schistosoma mansoni infection by temperature modulation. PLoS Pathog. 8(4): e1002677. doi:10.1371/journal.ppat.1002677

Lie, R.J., Heyneman, D., Richards, C.S., 1979. Specificity of natural resistance to trematode infections in Biomphalaria glabrata. Int. J. Parasitol. 9, 529-531.

Mascara, D., Kawano, T., Magnanelli, A.C., Silva, R.P.S., Sant'Anna, O.A., Morgante, J.S., 1999. Schistosoma mansoni: continuous variation in susceptibility of the vector snail of schistosomiais, Biomphalaria tenagophila I. Self-fertilization-lineage. Exp. Parasitol. 93, 133-141. 
Mitta, G., Adema, C.M., Gourbal, B., Loker, E.S., Theron, A., 2012. Compatibility polymorphism in snail/schistosome interactions: from field to theory to molecular mechanisms. Dev. Comp. Immunol. 37, 1-8.

Moné, Y., Gourbal, B., Duval, D., Du Pasquier, L., Kieffer-Jaquinod, S., Mitta, G., 2010. A Large Repertoire of Parasite Epitopes Matched by a Large Repertoire of Host Immune Receptors in an Invertebrate Host/Parasite Model. PLoS Negl. Trop. Dis. 4, e813.

Moné, Y., Ribou, A.C., Cosseau, C., Duval, D., Theron, A., Mitta, G., Gourbal, B., 2011. An exemple of molecular co-evolution: Reactive oxygen species (ROS) and ROS scavenger levels in Schistosoma mansoni/Biomphalaria glabrata interactions. Int. J. Parasitol. 41, 721-730.

Morand, S., Manning, S.D., Woolhouse, M.E.J., 1996. Parasite-host coevolution and geographic patterns of parasite infectivity and host susceptibility. Proc. R. Soc. Lond. B. 263, 119-128.

Morley, N.J., 2001. Inbred laboratory cultures and natural trematode transmission under climate change. Trends Parasitol. 27, 286-287.

Paraense, W. L., Corrêa, L.R., 1963. Variation in susceptibility of Australorbis glabratus to a strain of Schistosoma mansoni. Rev. Inst. Med. Trop. Sao Paulo. 5, 15-22.

Portela, J., Duval, D., Rognon, A., Galinier, R., Boissier, J., Coustau, C., Mitta, G., Théron, A., Gourbal, B., 2013. Evidence for specific genotype-dependent immune priming in the Lophotrochozoan Biomphalaria glabrata snail. J. Innate Immun. 5, 261-276.

Richards, C.S., 1970. Genetics of a molluscan vector of schistosomiasis. Nature. 227, 806810.

Richards, C.S., Shade P.C., 1987. The genetic variation of compatibility in Biomphalaria glabrata and Schistosoma mansoni. J. Parasit. 73, 1146-1151.

Richards, C.S., 1973. Susceptibility of adult Biomphalaria glabrata to Schistosoma mansoni infection. Am. J. Trop. Med. Hyg. 22, 748-756.

Richards, C. S. 1975. Genetic factors in susceptibility of Biomphalaria glabrata for different strains of Schistosoma mansoni. Parasitology. 70, 231-241.

Richards, C. S., Knight, M., Lewis, F. A., 1992. Genetics of Biomphalaria glabrata and its effect on the outcome of Schistosoma mansoni infection. Parasitol. Today. 8, 171-174.

Roger, E., Mitta, G., Moné, Y., Bouchut, A., Rognon, A., Grunau, C., Boissier, J., Théron, A., Gourbal, B.E., 2008a. Molecular determinants of compatibility polymorphism in the Biomphalaria glabrata/Schistosoma mansoni model: New candidates identified by a global comparative proteomics approach. Mol. Bio. Parasitol. 2, 205-216. 
Roger, E., Gourbal, B., Grunau C., Pierce R., Galinier R., Mitta G., 2008b. Expression analysis of highly polymorphic mucin proteins (Sm PoMuc) from the parasite Schistosoma mansoni. Mol. Bio. Parasitol. 2, 217-27.

Roger, E., Grunau, C., Pierce R.J., Hirai, H., Gourbal, B.E.F., Galinier, R., Emans, R., Cesari, I.M., Cosseau, C., Mitta, G., 2008c. Controlled chaos of polymorphic mucins in a metazoan parasite (Schistosoma mansoni) interacting with its invertebrate host (Biomphalaria glabrata). PloS. Negl. Trop. Dis. 2(11): e330.

Sire, C., Rognon, A., Theron, A., 1998. Failure of Schistosoma mansoni to reinfect Biomphalaria glabrata snails : acquired humoral resistance or intra-specific larval antagonism ? Parasitol. 117, 117-122.

Sturrock, R.F., 2001. Schistosomiasis epidemiology and control: how did we get here and where should we go ? Mem. Inst. Oswaldo Cruz. 26, 17-27.

Sullivan, J.T., Richards C.S., 1981. Schistosoma mansoni, NIH-SM-PR2 strain, in susceptible and nonsusceptible stocks of Biomphalaria glabrata: comparative histology. J. Parasitol. 5, 702-708.

Theron, A., Gerard, C., 1994. Development of accessory sexual organs in Biomphalaria glabrata as related to infection timing by Schistosoma mansoni: Consequences on the energy utilisation patterns by the parasite. J. Moll. Stud. 60, 78-85.

Theron, A., Pages, J. R., Rognon, A., 1997. Schistosoma mansoni: distribution patterns of miracidia among Biomphalaria glabrata snail as related to host susceptibility and sporocyst regulatory processes. Exp. Parasitol. 85, 1-9.

Theron, A., Coustau, C., 2005. Are Biomphalaria snails resistant to Schistosoma mansoni ? J. Helminthol. 79, 1-6.

Theron, A., Coustau, C., Rognon, A., Gourbière, S., Blouin, M.S., 2008. Effects of laboratory culture on compatibility between snails and schistosomes. Parasitology. 135, 11791188 .

Vasquez, R.E., Sullivan, J.T., 2001. Further characterization of passively transferred resistance to Schistosoma mansoni in the snail intermediate host Biomphalaria glabrata. J. Parasitol. 6, 1360-1365.

Webster, J. P., Gower, C. M., Blair, L., 2004. Do hosts and parasites coevolve? Empirical support from the Schistosoma system. American Naturalist 164 Suppl 5, S33-51.

Webster, J. P., Woolhouse, M.E.J., 1998. Selection and strain specificity of compatibility between snail intermediate hosts and their parasitic schistosomes. Evolution Int. J. Org. Evolution. 52, 1627-1634. 
Webster, J.P., Davies, C.M., 2001. Coevolution and compatibility in the snail-schistosome system. Parasitology. 123, S41-S56.

WHO. 2010. World Health Organisation. Schistosomiasis, Fact Sheet No 115.

Yoshino, TP., Coustau, C., 2011. Immunobiology of Biomphalaria glabrata-trematode interactions, in:. Toledo, R., Fried, B. (Eds), Biomphalaria Snails and larval trematodes. Springer Science+Business Media, pp.159-189. 


\section{Legends to Figures}

Fig. 1 - Infection rates $(\% \pm 1$ standart error) of individual snails of the five Biomphalaria glabrata strains (BgBRE, BgVEN, BgGUA, BgBAR and BgBS-90) exposed to increasing doses of miracidia (Mi) from the four strains of Schistosoma mansoni (SmBRE, SmGUA, SmVEN and SmLE).

Fig. 2 - Infectivity (\% of infected snails \pm 1 standart error) of four strains of Schistosoma mansoni (SmBRE, SmGUA, SmVEN and SmLE) towards the five Biomphalaria glabrata host strains (BgBRE, BgVEN, BgGUA, BgBAR and BgBS-90) exposed to increasing doses of miracidia (Mi).

Fig. 3 - Evolution of parasite intensities (mean number of mother sporocysts (Msp) per infected snails \pm 1 standart error): A) after exposure of BgBRE snails to increasing doses of miracidia (Mi) from the four strains of Schistosoma mansoni (SmBRE, SmGUA, SmVEN and SmLE); B) after exposure of Biomphalaria glabrata snails from the five strains (BgBRE, BgVEN, BgGUA, BgBAR and BgBS-90) with increasing doses of miracidia (Mi) from the SmBRE strains of Schistosoma mansoni. Percentages in brackets correspond to infection rates of the host-parasite combinaison for a dose of $50 \mathrm{Mi} / \mathrm{snail}$.

Fig. 4 - Average transformation rates of miracidia into mother sporocysts for the different doses of miracidia (Mi) at exposure time: A) for each schistosome strains, all host strains included; B) for each host strains, all schistosome strains included. Dashed lines represent the total average for each strain including all miracidial doses (except for $1 \mathrm{Mi}$ ).

Fig. 5 - Radar graph representation of the multi-parasite susceptibility phenotypes characterizing each Biomphalaria glabrata strain (BgBS-90, BgBAR, BgBRE, BgGUA and BgVEN) vis-à-vis of the four strains of Schistosoma mansoni (SmLE, SmVEN, SmBRE and SmGUA). Infection rate (\%) and parasite intensity (number of mother sporocysts (Msp) per snail) values are those corresponding to an exposure dose of 20 miracidia/snail. 
Fig. 6 - Radar graph representation of the multi-host infectivity phenotypes characterizing each strain of Schistosoma mansoni (SmBRE, SmGUA, SmVEN and SmLE) vis-à-vis of the five Biomphalaria glabrata strains (BgBS-90, BgBAR, BgBRE, BgGUA and BgVEN). Infection rate (\%) and parasite intensity (number of mother sporocysts (Msp) per snail) values are those corresponding to an exposure dose of 20 miracidia/snail.

Fig. 7 - Selection patterns on each Biomphalaria glabrata strain, to create new discreet host phenotypes: (A) from the BgBs90 strain, un-susceptible to 4 Schistosoma mansoni strains (SmBRE, SmGUA, SmVEN and SmLE); (B) from the BgBAR strain, un-susceptible to 3 parasite strains (SmBRE, SmGUA and SmVEN); (C) from the BgGUA and BgVEN strains, un-susceptible to 2 parasite strains (SmBRE and SmGUA); (D) from the BgBRE strain, unsusceptible to one parasite strain (SmGUA); (E) from the BgVEN and BgGUA strains, susceptible to 4 parasite strains ( see text for more details). 

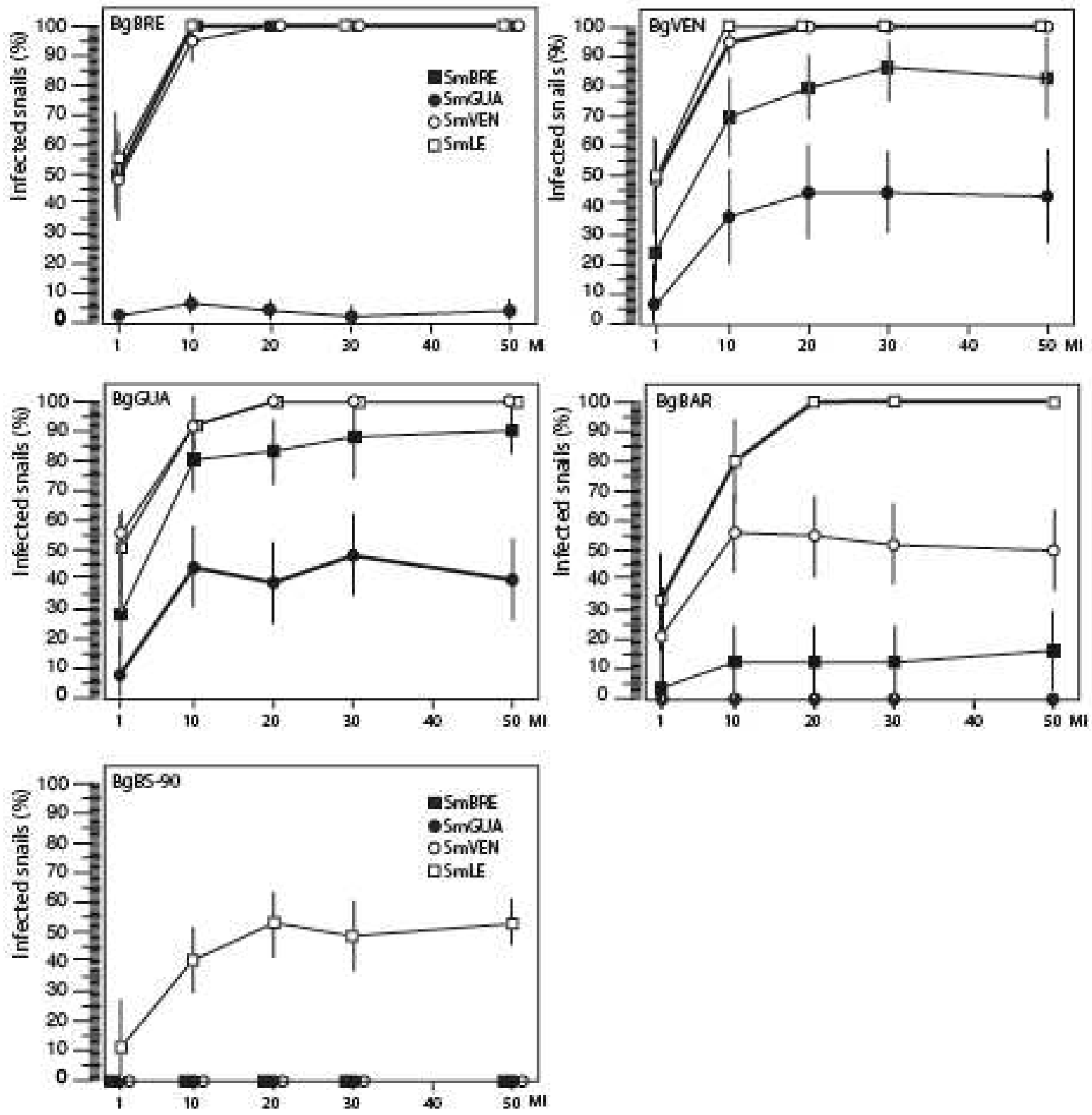

Fig. 1 

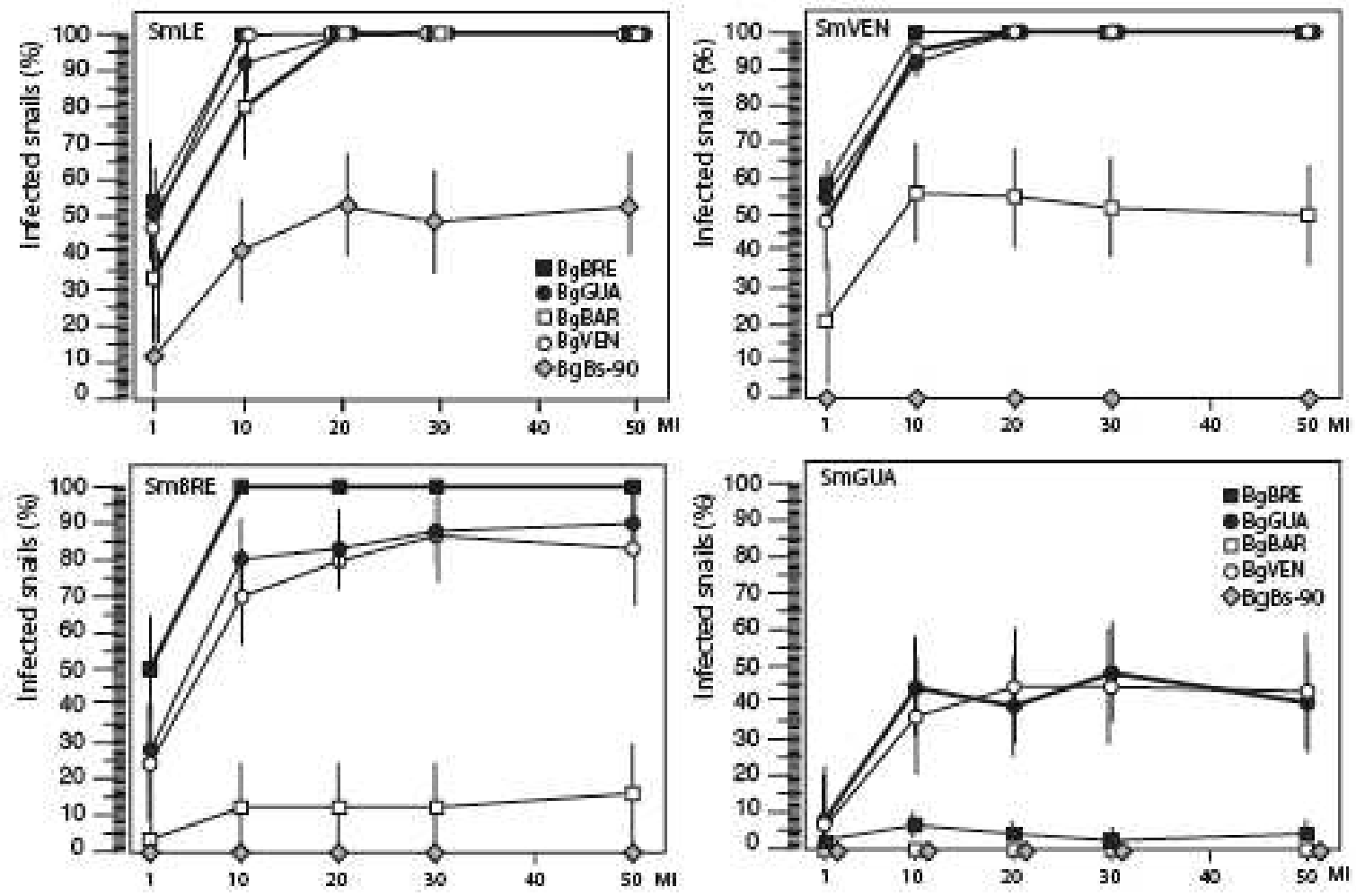

Fig. 2 

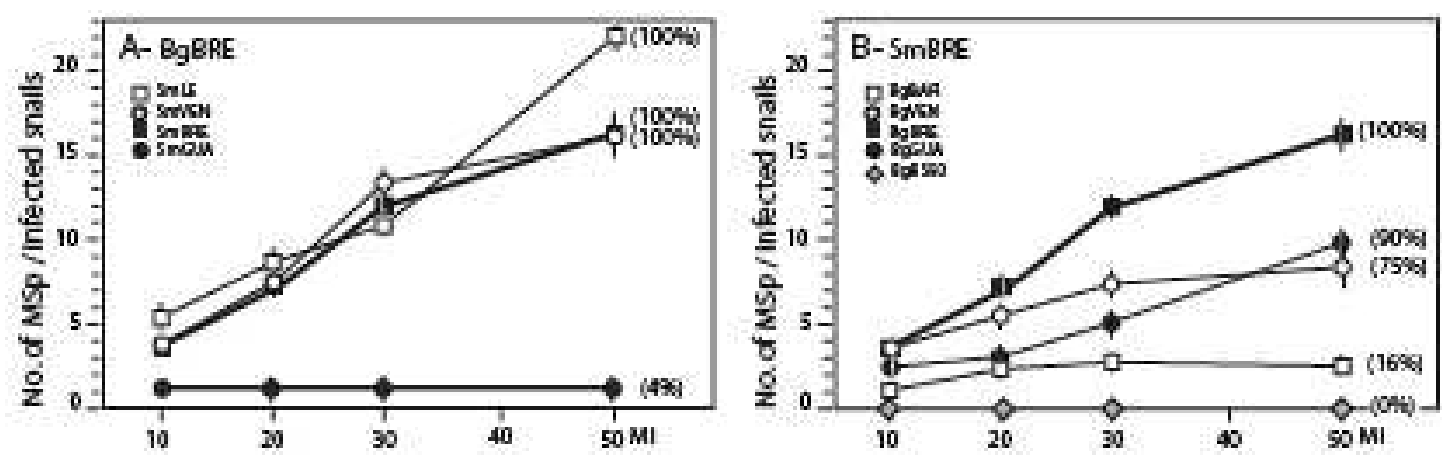

Fig. 3 


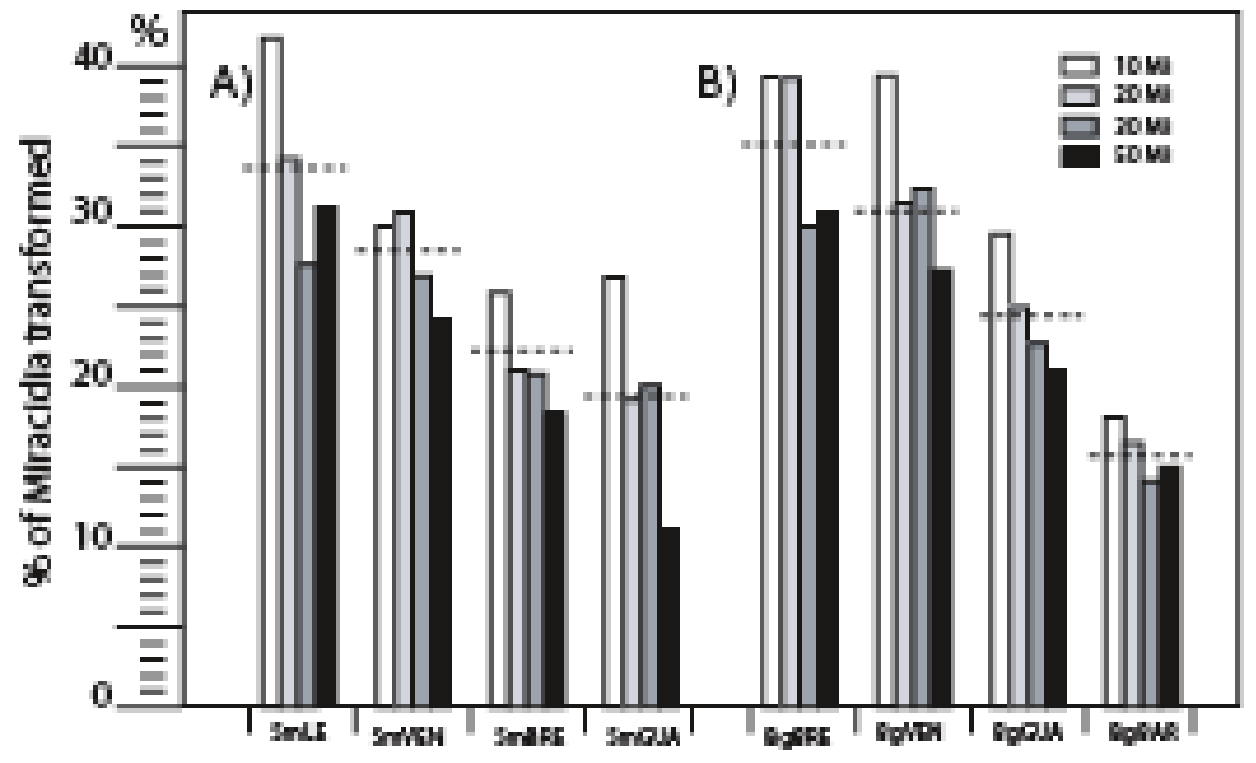

Fig. 4 

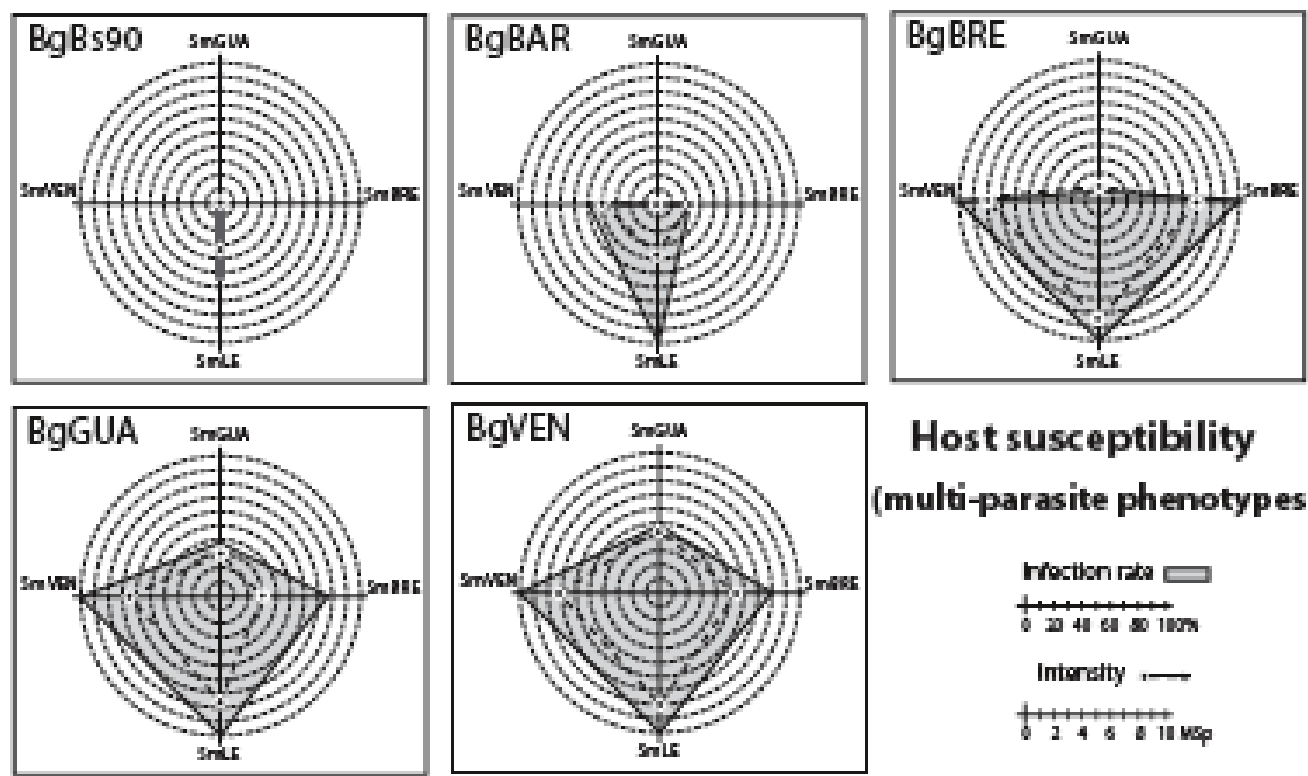

Host susceptibility (multi-parasite phenotypes)

rroction reso $\square$

०ै

Intaratty -

Fig. 5 

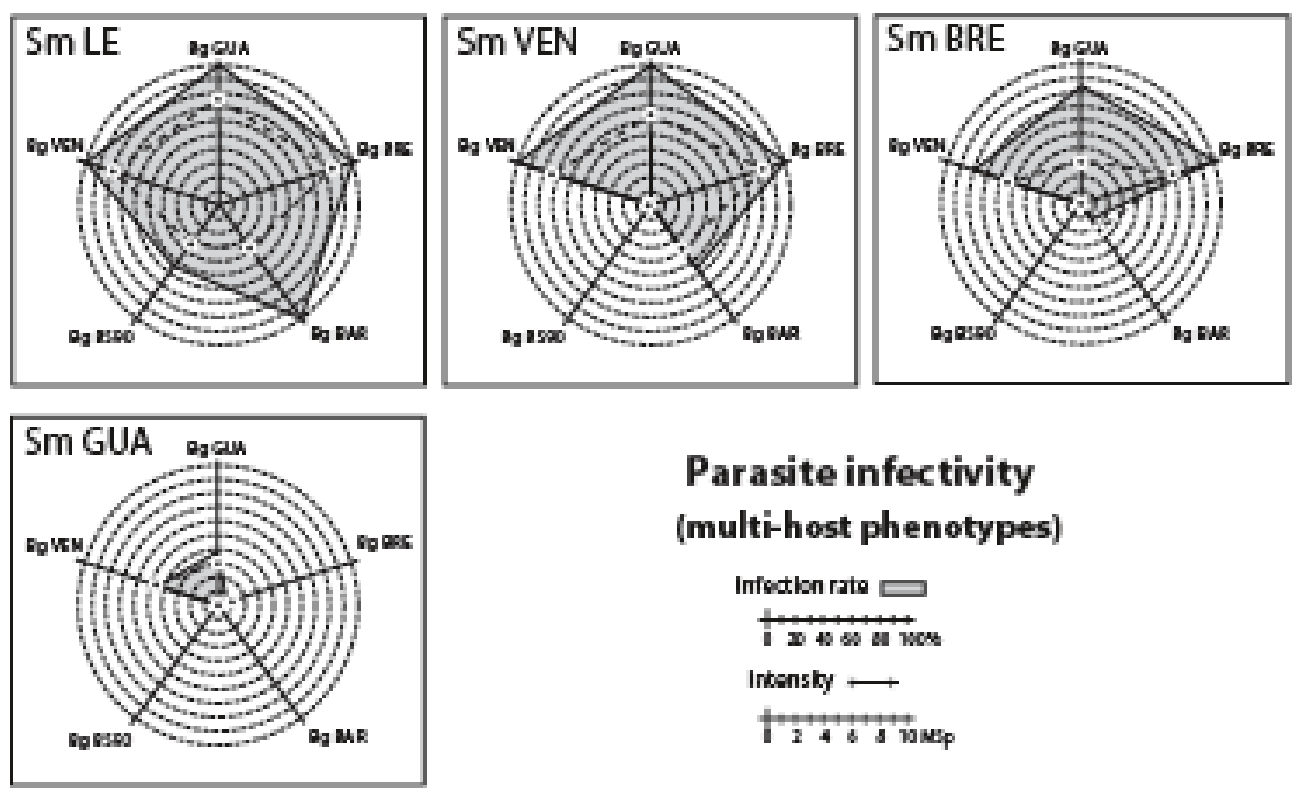

Fig. 6
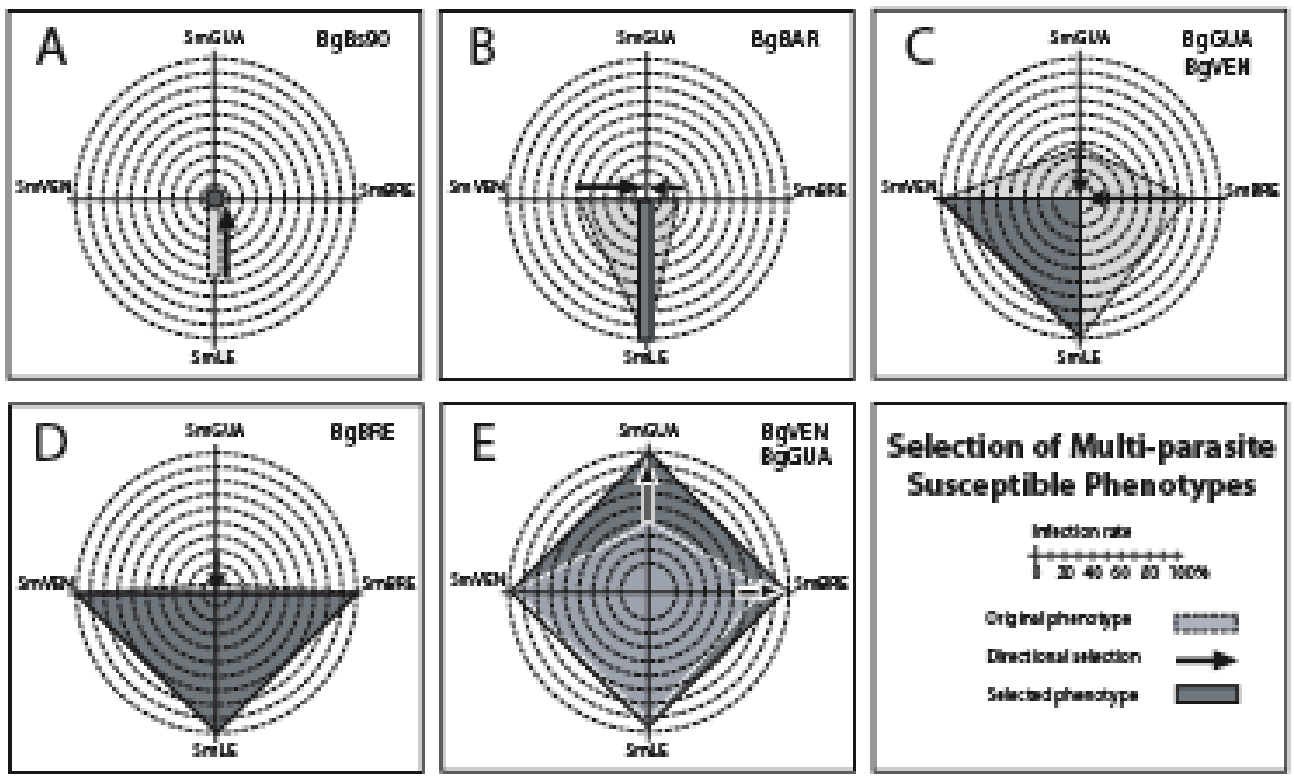

Fig. 8 